\title{
Optimization of Banana Peels Hydrolysis for the Production of Bioethanol: Response Surface Methodology
}

\author{
Mebrahtom Gebresemati ${ }^{1, a *}$ and Alula Gebregergs ${ }^{2, b}$ \\ ${ }^{1,2}$ Department of Biological and Chemical Engineering, MIT Mekelle University, Mekelle, Ethiopia \\ amebreamit@gmail.com, balula.gebregergs@gmail.com
}

Keywords: Bioethanol, Banana peel, Hydrolysis, Fermentation, Sugar content

\begin{abstract}
Energy consumption has increased steadily over the last four decades as the population has grown and more countries have become industrialized. On the other hand waste disposal has become the major concern of developing cities. Many countries such as Ethiopia have abundant raw materials for biofuels, yet these have not been explored. This study was designed to utilize banana peels for the production of bioethanol using the yeast Saccharomyces cerevisiae. The effects of factors in hydrolysis (the effect of hydrolysis parameters) were investigated and the optimum combination factor was carried out with response surface design. The parameters were varied over 3 levels and 17 experimental runs were conducted to produce fermentable sugar. The optimum results were obtained at $1.50 \% \mathrm{v} / \mathrm{v}$ acid concentration, $91.02{ }^{\circ} \mathrm{C}$ temperature and 21.66 min retention time. At this optimum condition, fermentation with and without benzyl penicillin was performed to determine its effect on bioethanol.
\end{abstract}

\section{INTRODUCTION}

The overall well-being of the world; industrial competitiveness and the functioning of society are dependent on safe, sustainable and affordable energy. Energy provides an essential power for almost all human activities: it provides services for cooking, heating, lighting, health, food production and storage, education, mineral extraction, industrial production and transportation [1]. Even though, world primary energy source nowadays is dominated by fossil fuels (coal, oil and natural gas), the acceleration of technological development has opened door to continuous and rapid worldwide economic growth and in fact allowed the world to achieve energy sustainability using many different energy sources called renewable energy sources, comprising mainly biofuels, hydropower, geothermal, wind and solar energy, currently represent less percentage of the primary energy use [1]

Energy consumption has increased steadily over the last century as the world population has grown and more countries have become industrialized. Crude oil has been the major resource to meet the increased energy demand [1,2]. Different techniques has been used to estimate the current known crude oil reserves and concluded that the annual global oil production would decline from the current 25 billion barrels to approximately 5 billion barrels in 2050 [2].

Ethanol (ethyl alcohol, bioethanol) is the most employed liquid biofuels either as a fuel or as a gasoline enhancer [3]. Ethanol has some advantages when it is used as an oxygenate. Firstly, it has a higher oxygen content that implies a less amount of required additive. The increased percentage of oxygen allows a better oxidation of the gasoline hydrocarbons with the consequent reduction in the emission of $\mathrm{CO}$ and aromatic compounds [3,4]. Related to MTBE, ethanol has greater octane booster properties, it is not toxic, and does not contaminate water sources. Nevertheless, ethanol production costs are higher than those of MTBE, gasoline mixed with alcohol conduces the electricity, and Reid vapor pressure is higher that entails a greater volatilization, which can contribute to ozone and smog formation. Many countries have implemented or are implementing programs for addition of ethanol to gasoline [3].

The fuel ethanol can be obtained from energy crops and lignocellulosic biomass. The complexity of the production process depends on the feedstock. In this way, the spectrum of designed and implemented technologies goes from the simple conversion of sugars by fermentation, to the multistage conversion of lignocellulosic biomass into ethanol [5]. 
Different biofuels such as ethanol, methanol, bio-diesels, etc. are produced by fermentation of agricultural wastes, fruit wastes, municipal and industrial wastes using Saccharomyces cerevisiae (baker's yeast) as food for the microorganisms. Amongst these biofuels, ethanol has great demand as it is widely accepted and it is clean burning [3,5]. A treatment of municipal solid waste goes far back into 18th century when burying the waste was the best option of management. In course of time, the scarcity of resources and the soaring pollution level necessitated the need for alternative treatment options [6]. Recently, there are many treatment options for municipal solid waste among which composting, incineration, land filling and production of different biofuels are some. The choice among these options must be based on different technical and economic criteria of the situation in question $[5,6]$.

In most developing African countries, municipal solid wastes are disposed of in nonengineered landfills which have always been known to generate greenhouse gases. In developed nations, conversely, municipal solid wastes are treated through such advanced methods as controlled incineration and production of biofuels because of both increasing need of using the so called "waste" as resource and stringent environmental regulations [6].

On the other hand inadequate municipal and industrial solid waste collection and disposal creates a range of environmental problems in our country. A considerable amount of waste ends up in open dumps or drainage system, threatening both surface water and ground water quality and causing serious of environmental and health problems. Open air burning of waste, spontaneous combustion in landfills and incinerating plants that lack effective treatment for gas emissions are causing air pollution [6]. The adverse effects of inadequate solid waste service create significant effect on productivity and economic development. Solid waste such as fruit peels largely obtained as a byproduct from food processing industries, juice processing houses, hotels and restaurants in our country. These wastes can entail serious environmental problems unless they are changed in to some useful products or disposed properly $[5,6]$.

The aim of study was to determine optimum parameters (acid concentration, temperature and time) for hydrolyzing waste banana peels to obtain maximum amount of fermentable sugars by conducting series of experimental analysis. The effect of benzyl penicillin was also studied during fermentation.

\section{MATERIAL AND METHODS}

\subsection{Material}

2.1.1 Raw material: Banana peels were collected in plastic bags from juice houses.

2.1.2 Chemicals: $98 \%$ sulfuric acid $\left(\mathrm{H}_{2} \mathrm{SO}_{4}\right)$, Sodium hydroxide $(\mathrm{NaOH})$, Yeast extracts agar, Urea, Dextrose sugar, $\mathrm{MgSO}_{4} \cdot 7 \mathrm{H}_{2} \mathrm{O}$, Baker yeast/Yeast (Saccharomyces cerevisiae), Distilled water, Benzathine penicillin G.

2.1.3 Equipment: Plastic bags, knife, oven, grinder, electronic balance, digital $\mathrm{pH}$ meter, flasks, graduated cylinders of different volumes, autoclave, filter, shaking incubator, rotary evaporator, pycnometer.

\subsection{Method: Box-Behnken Experimental Design}

2.2.1 Feed Material: The gathered peels were chopped into small pieces about $2-4 \mathrm{~cm}$ length using knife. The pieces were then sun dried under mild sunlight for two days and finally dried at $60{ }^{0} \mathrm{C}$ in oven for one day. Then the samples were taken out of the drier when they were dried enough to be crushed. The cut pieces were then crushed in the mixer grinder. The ground sample was proportioned into seventeen separate samples of $10 \mathrm{~g}$ of ground banana peels and another two separate samples of $20 \mathrm{~g}$ of ground banana peels. For all the samples juice were prepared, by adding 10:1(v/w) ratio of distilled water to the sample in separate flasks.

2.2.2 Steam Treatment: The separate samples capped with aluminum foil were autoclaved at $15 \mathrm{psi}$ pressure for $30 \mathrm{~min}$. After autoclaving the samples was allowed to cool and the soluble portion was separated from the insoluble by using filtration. Next, the insoluble portion was hydrolyzed in the next step and amount of sugar produced was measured for each sample. 
2.2.3 Acid Hydrolysis: First $25 \mathrm{ml}$ of $0.5 \%$ to $2.5 \%(\mathrm{v} / \mathrm{v})$ diluted sulfuric acid was added to the insoluble component from pretreatment steps. The fruit peels were then hydrolyzed in the reactor between 70 and $110{ }^{\circ} \mathrm{C}$ for 10 to $30 \mathrm{~min}$ as the experimental design order (Table 1). After hydrolysis, neutralization was carried out with $1 \mathrm{M} \mathrm{NaOH}$ until the $\mathrm{pH}$ became around 7 . Insoluble particles were separated from the hydrolysate by filtration (to remove the non-fermentable lignin portion). Then the soluble component was added with the previously filtered solution from the pretreatment step for the next procedure.

Table 1: Experimental design formulation for hydrolysis stage

\begin{tabular}{|l|l|l|l|}
\hline Run & $\begin{array}{l}\text { Acid conc. [\% v/v to } \\
\text { dist. Water] }\end{array}$ & $\begin{array}{l}\text { Hydrolysis } \\
\text { temp. }\left[{ }^{0} \mathrm{C}\right]\end{array}$ & $\begin{array}{l}\text { Hydrolysis } \\
\text { time [min }]\end{array}$ \\
\hline 1 & 2.5 & 90 & 30 \\
\hline 2 & 1.5 & 90 & 20 \\
\hline 3 & 2.5 & 70 & 20 \\
\hline 4 & 1.5 & 90 & 20 \\
\hline 5 & 0.5 & 110 & 20 \\
\hline 6 & 2.5 & 90 & 10 \\
\hline 7 & 1.5 & 90 & 20 \\
\hline 8 & 2.5 & 110 & 20 \\
\hline 9 & 1.5 & 90 & 20 \\
\hline 10 & 1.5 & 90 & 20 \\
\hline 11 & 1.5 & 110 & 10 \\
\hline 12 & 0.5 & 70 & 20 \\
\hline 13 & 0.5 & 90 & 30 \\
\hline 14 & 0.5 & 90 & 10 \\
\hline 15 & 1.5 & 70 & 10 \\
\hline 16 & 1.5 & 70 & 30 \\
\hline 17 & 1.5 & 110 & 30 \\
\hline
\end{tabular}

2.2.4 Measuring Sugar Content: Brix method was used to measure the sugar contents of each sample. First, the weight of an empty dry flask equal with the sample flask was weighed. Next, the weight of the flask with distilled water up to the level of the juice on the sample flask was weighed. The procedure was repeated for each flask with the juice. Finally, the maximum sugar content percentage was calculated and the parameters condition (acid concentration, temperature, and time) that gave rise to it was taken as an optimum condition. Using this optimum condition, hydrolysis was carried out for the two samples containing $20 \mathrm{~g}$ banana peel powder. Once again the lignin was removed from the soluble portion and the soluble solution found was added into the previously separated soluble portions. Finally, these two samples were used to produce ethanol from banana peels by the Saccharomyces Cerevisiae.

2.2.5 Fermentation: Media was prepared for fermentation process; fermentation with $S$. cerevisiae only and fermentation with $S$. cerevisiae and benzathine penicillin G. $100 \mathrm{ml}$ from the inoculum was added to $0.5 \mathrm{~g}$ of yeast ( $5 \mathrm{~g} / \mathrm{L}$ yeast), $S$. cerevisiae, in $250 \mathrm{ml}$ flask. The flask was properly covered with aluminum foil to prevent air entrance (the growth was made anaerobically). The conical flask was then placed in a shaking incubator for 24 hours at temperature of $30{ }^{0} \mathrm{C}$ and $200 \mathrm{rpm}$, which was used for the next process of fermentation. In the fermentation step, the two samples were conditioned to a temperature of $30{ }^{0} \mathrm{C}$ before fermentation step started. This was the working temperature throughout the whole fermentation process. At this step the $\mathrm{pH}$ was set to 5.05.5 , optimum $\mathrm{pH}$ for the activity of $S$. cerevisiae by the addition of $1 \mathrm{M} \mathrm{NaOH}$. Then $25 \mathrm{ml}$ freshly prepared yeast culture was added into each of the flasks $(1: 4(\mathrm{v} / \mathrm{v})$ of samples $)$ and the flasks mouth was raped with aluminum foil. Next, $0.05 \mathrm{~g}$ of benzathine penicillin $\mathrm{G}(0.5 \mathrm{~g} / \mathrm{L})$ was added into the second flask while the first remains as it is. Finally, both of the samples were placed in shaker 
incubator at $200 \mathrm{rpm}, 30{ }^{\circ} \mathrm{C}$, for 3 days. The samples were checked every day by adjusting the $\mathrm{pH}$ set between 5.0 and 5.5 .

2.2.6 Distillation and Density Measurement: In the production of ethanol from the banana peel juice rotary evaporator and simple distillation were used to get distillate product. The final products were evaluated for their ethanol content by measuring the corresponding density using pycnometer at room temperature. The specific gravity of the produced alcohol was determined and alcohol concentration was got from the relationship between the specific gravity and the proportion of ethanol in alcohol solution at room temperature. Specific gravity of sample was calculated according to the formula given below.

Specific gravity of sample $=\left(\mathrm{W}_{2^{-}}-\mathrm{W}_{1}\right) /\left(\mathrm{W}_{3}-\mathrm{W}_{1}\right)$, where $\mathrm{W}_{1}=$ weight of empty pycnometer, $\mathrm{W}_{2}=$ weight of pycnometer plus sample, and $\mathrm{W}_{3}=$ weight of pycnometer filled with distilled water.

\section{RESULTS AND DISCUSSION}

3.1 Normal Probability and Predicted Response Model: To determine the model satisfies the assumptions of the analysis of variance (ANOVA), the normal plot with residue and the plots of residuals versus predicted were analyzed, which is shown in Fig. 1 and Fig 2. In normal probability plot of the raw data the analysis of variance, shows more effective (straight forward) with the residuals. The quadratic polynomial model satisfies the assumptions analysis of variance (ANOVA) i.e. the error distribution is approximately normal (Fig.1). If the model is correct and the assumptions are satisfied, the residuals should be structure less; in particular, they should be unrelated to any other variable including the predicted response. A simple check is to plot the residuals versus the fitted (predicted) values. A plot of the residuals versus the rising predicted response values tests the assumption of constant variance. The plot shows random scatter which justifying no need for an alteration to minimize personal error (Fig.2).

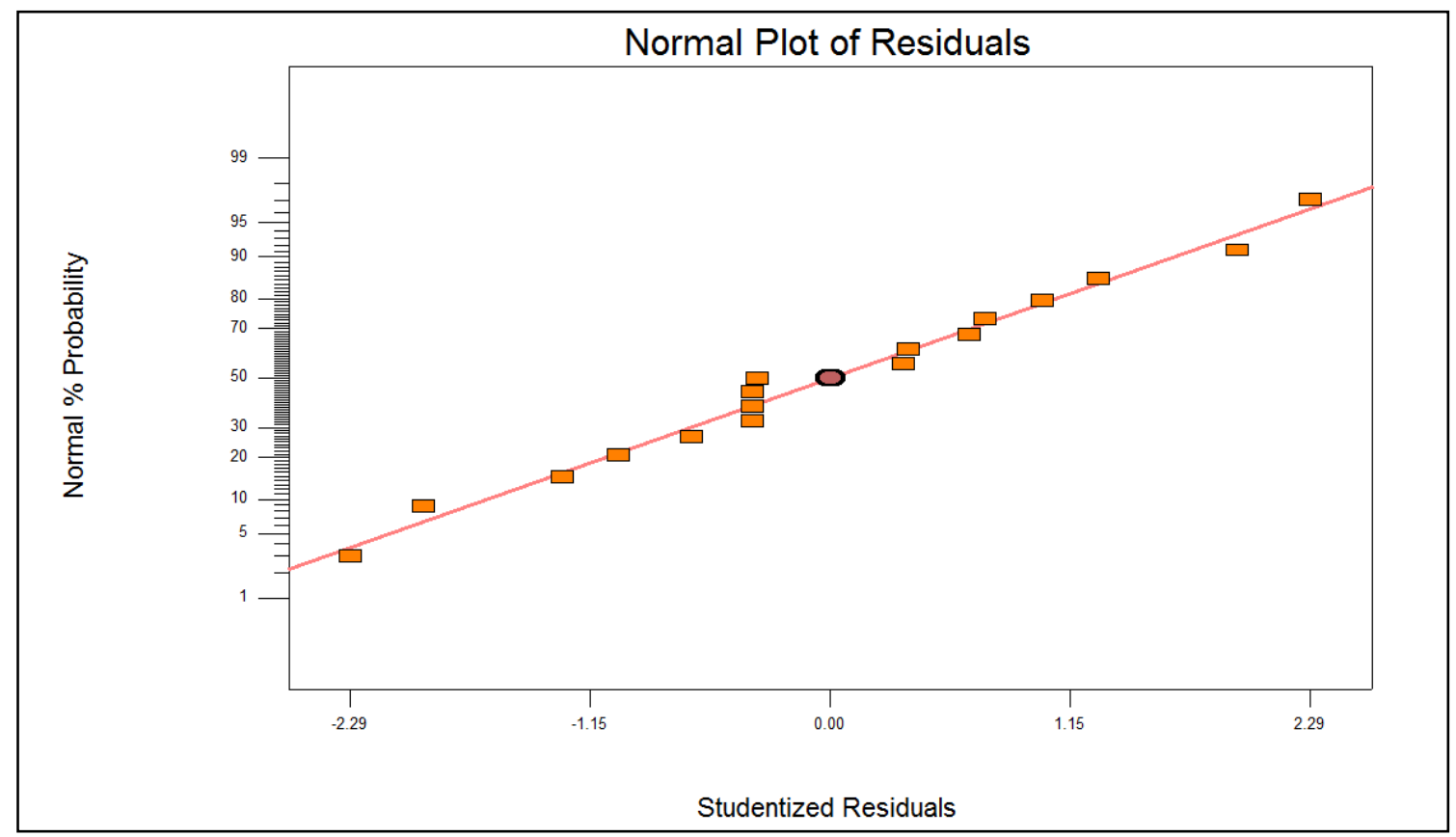

Fig. 1: Normal plots of residuals 


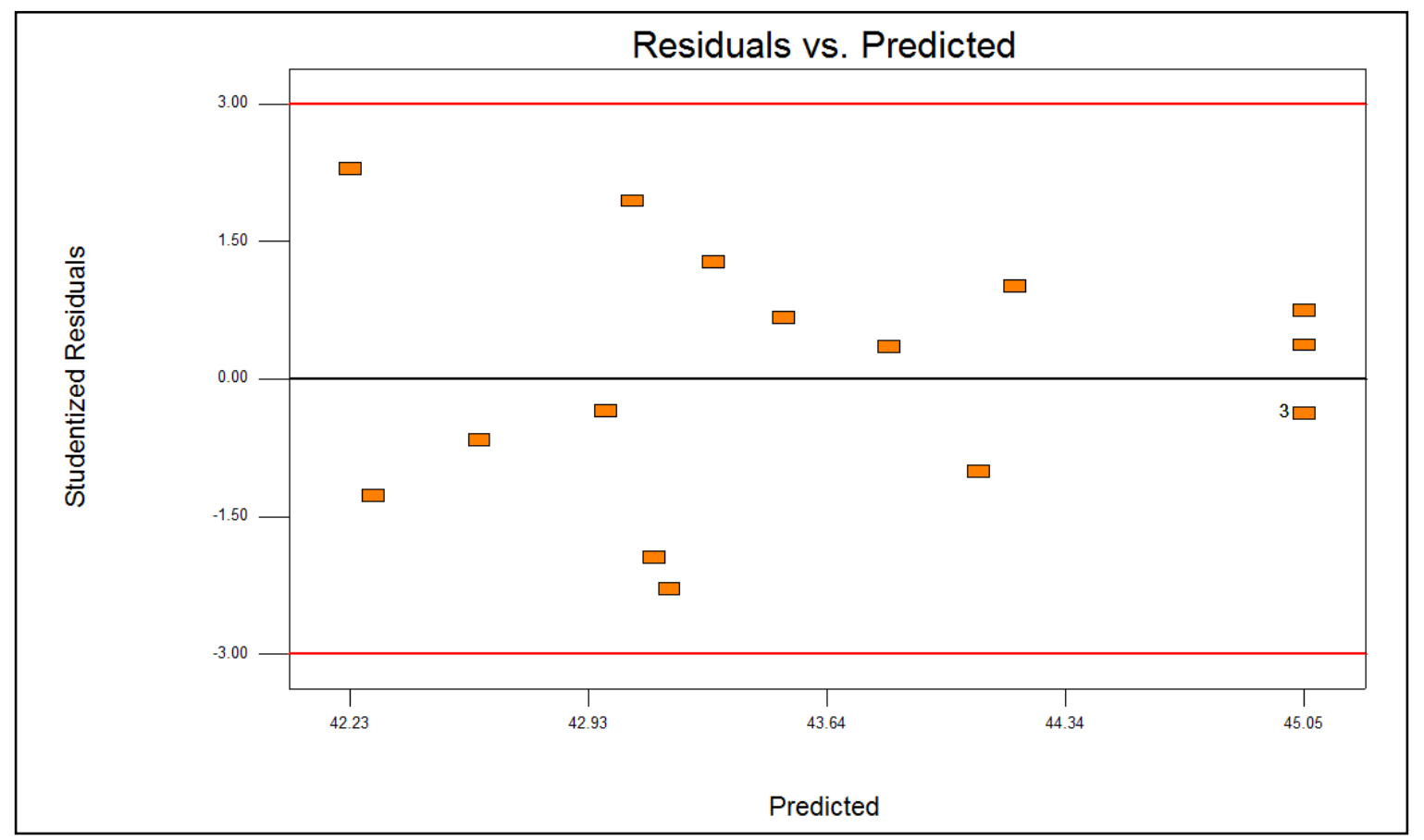

Fig.2: Residual vs. predicted values

3.2 Effects of Parameters on Hydrolysis: The effects of acid concentration and time, effects of acid concentration and temperature, and effect of temperature and time on hydrolysis for the yield of fermentable sugar were represented by three dimensional response surface plots shown in Fig. 3, 4, \& 5, respectively.

The response surface obtained from acid concentration and hydrolysis time (Fig. 3), conical shape, defined the optimum operating conditions. As hydrolysis time increases at lower level of acid concentration, and as increase level of acid concentration and lower level of time gives a positive effect on the yield of sugar. The response surface also suggests that there were dominance interactions of these two factors.

The effect of acid concentration and temperature on the yield of sugar is shown in Fig. 4. It was observed that when the levels of temperature increase hydrolysis results high yield of sugars. However from the graph after some increments of temperature, the yield of sugar decreases, this might be due to the possible formation of other molecules instead of glucose due to high temperature [7].

The response surface represented in Fig. 5, which obtained from hydrolysis temperature and hydrolysis time, conical shape, was observed, and the response optimized value for the production of sugar was based on both hydrolysis temperature and time. The response revealed that when levels of temperature increase hydrolysis results high yield of sugars. However from the graph after some increments of temperature, the yield of sugar decreases. Similarly, at short and long time duration, the yield of sugar decrease. 


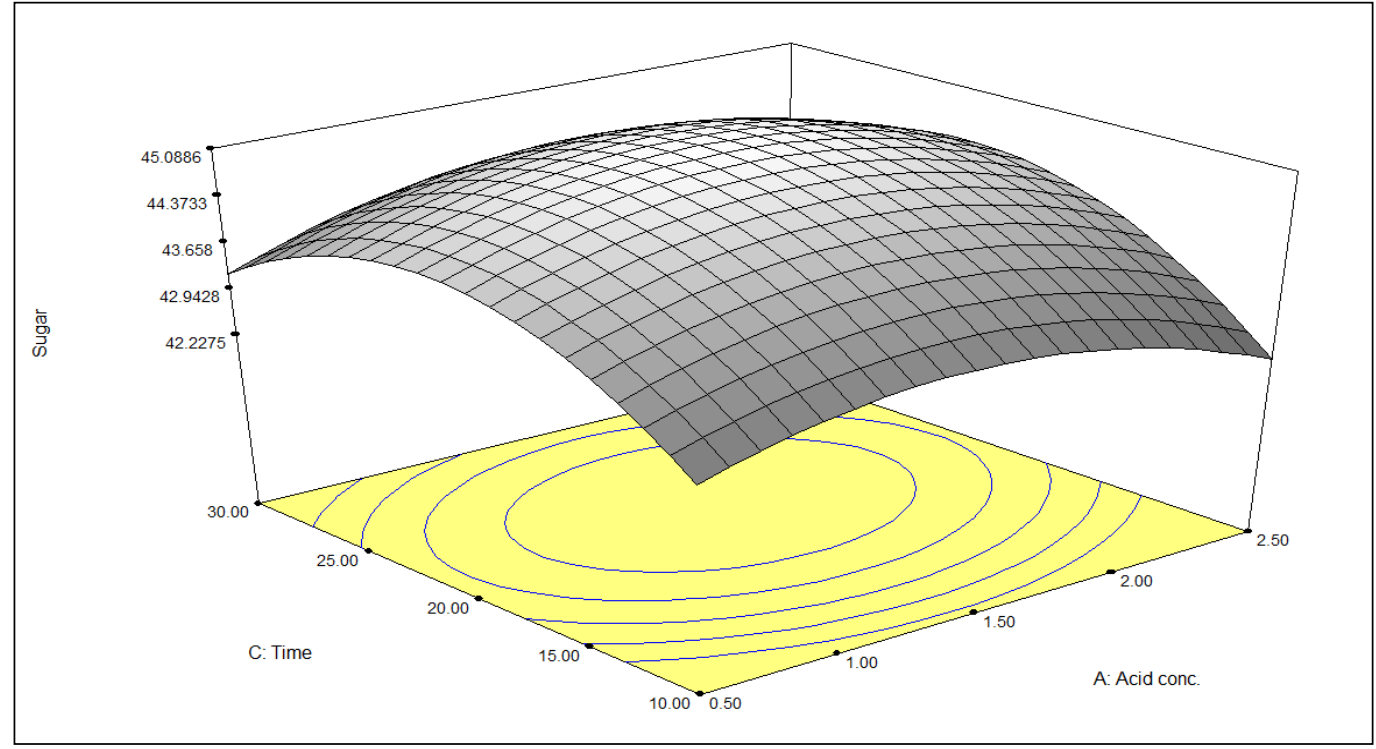

Fig. 3: Effect of acid concentration and time on the yield of fermentable sugars when temperature was at the center point.

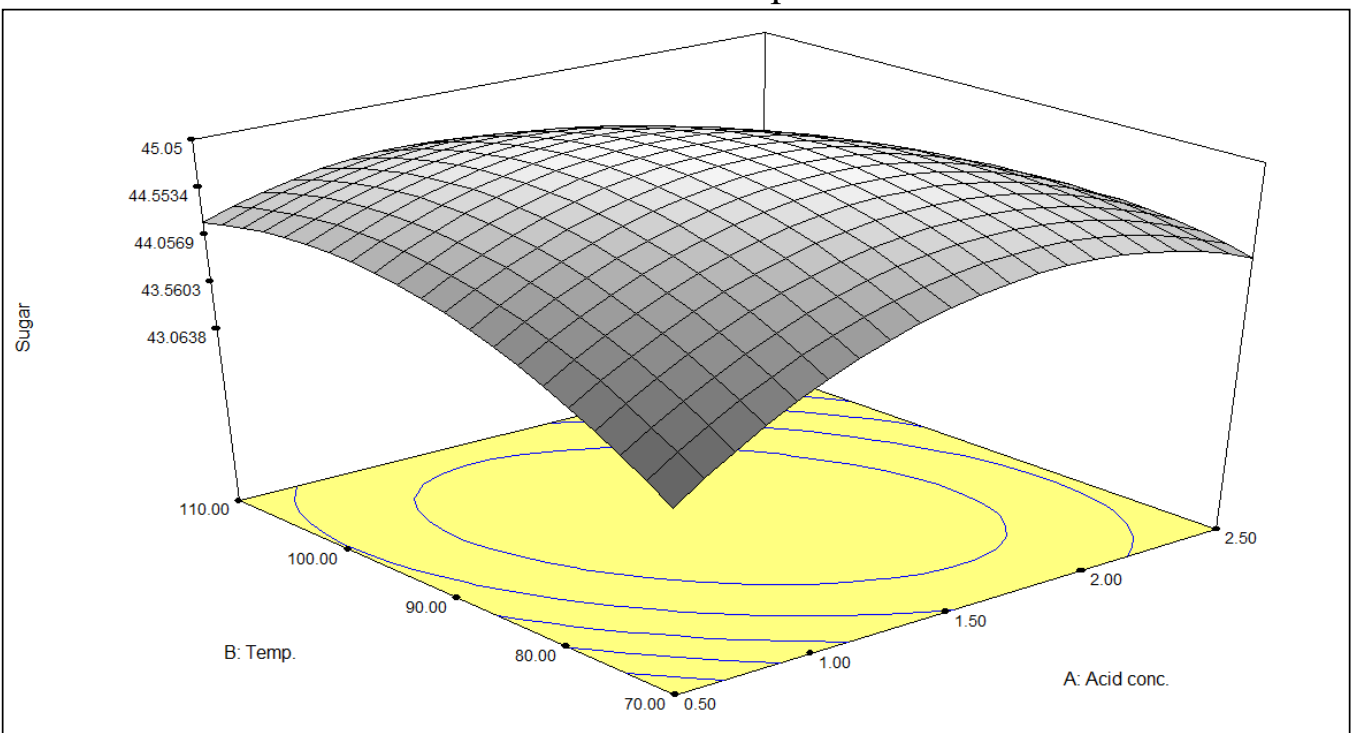

Fig. 4: Effect of acid concentration and temperature on the yield of fermentable sugars when time was at the center point.

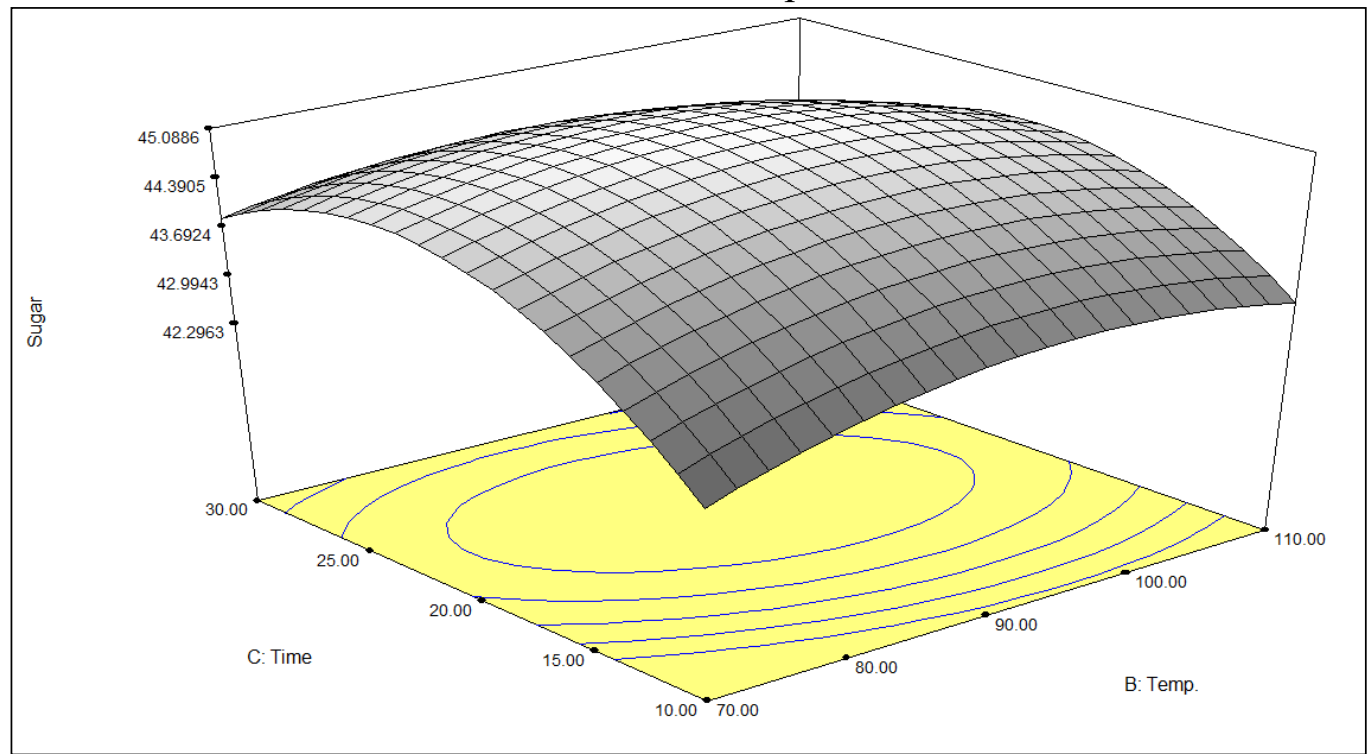

Fig. 5: Effect of temperature and time on the yield of fermentable sugars when acid concentration was at the center point. 
3.3 Optimization: The optimization was carried out with time, temperature and acid concentration. The numerical optimization of hydrolysis criteria for ethanol production from banana peel using dilute acid treatment are summarized in Table 2. The optimum possible solutions in hydrolysis for different parameters for ethanol production are expressed in Table 3.

Table 2: Optimization criteria for optimum yield of soluble sugar content

\begin{tabular}{|l|l|l|l|}
\hline Constraints & \multicolumn{3}{|l|}{} \\
\hline Name & Goal & Lower Limit & Upper Limit \\
\hline Acid conc. & is in range & 0.5 & 2.5 \\
\hline Temp. & is in range & 70 & 110 \\
\hline Time & is in range & 10 & 30 \\
\hline Sugar & maximize & 42.20 & 45.05 \\
\hline
\end{tabular}

Table 3: Optimum possible solutions (10 solutions found)

\begin{tabular}{|l|l|l|l|l|l|}
\hline Number & Acid conc. & Temp. & Time & Sugar & Desirability \\
\hline $\mathbf{1}$ & $\mathbf{1 . 5 0}$ & $\mathbf{9 1 . 0 2}$ & $\mathbf{2 1 . 6 6}$ & $\mathbf{4 5 . 0 8 8}$ & $\mathbf{1}$ (Selected) \\
\hline 2 & 1.43 & 87.55 & 22.05 & 45.070 & 1 \\
\hline 3 & 1.60 & 86.97 & 23.02 & 45.064 & 1 \\
\hline 4 & 1.74 & 86.8 & 22.10 & 45.055 & 1 \\
\hline 5 & 1.31 & 89.76 & 20.93 & 45.050 & 1 \\
\hline 6 & 1.57 & 94.03 & 22.04 & 45.051 & 1 \\
\hline 7 & 1.51 & 92.51 & 21.62 & 45.079 & 1 \\
\hline 8 & 1.40 & 93.81 & 22.29 & 45.057 & 1 \\
\hline 9 & 1.31 & 92.42 & 21.53 & 45.059 & 1 \\
\hline 10 & 1.51 & 85.73 & 21.45 & 45.063 & 1 \\
\hline
\end{tabular}

3.4 Effect of Benzathine Penicillin G: the effect of benzathine was carried out by measuring the specific gravity of produced ethanol.

- $\quad$ Specific gravity of sample one $=(48.100-25.100) /(48.500-25.100)=0.98291$

- $\quad$ Specific gravity of sample two $=(48.073-25.100) /(48.500-25.100)=0.98175$

From the calculation it was found that the addition of benzathine penicillin $G$ enhanced the fermentation process by $8.97 \%$. In open literature this kind of work was not found. One of the author Barber et al. (2002) [8] has investigated ethanol production potential of local yeast strains isolated from ripe banana peels, and he found that Saccharomyces kluvveri $K-6$ ferment $40 \%(\mathrm{v} / \mathrm{v})$ of the glucose at $30{ }^{0} \mathrm{C}$ to yield $3.6-5.8 \%$ ethanol. Hence, the experimental result found to be impressive.

\section{CONCLUSIONS}

Hydrolysis of banana peel waste was carried out with dilute sulfuric acid with the objective to determine the optimum operating conditions that yield maximum sugar concentration. The experiment was designed by Box-Behnken response surface method. The optimum conditions (acid concentration, temperature and time) were examined by plotting the interaction, contour and 3D plots. Those all three factors were significant variables for the yield of optimum fermentable sugars. Very high and low acid concentration, temperature and hydrolysis time have negative effect on the yield of sugars. In addition to this the effect of benzathine penicillin $\mathrm{G}$ on fermentation process was examined by measuring the specific gravity of the produced ethanol. The presence of benzathine penicillin $\mathrm{G}$ had enhanced the final ethanol production. 


\section{Acknowledgements}

The authors wish to thank Mr Abadi Gebreyesus, Mr Hailekiros Tadesse and Mr Kibrom Gebreegzabher for their efforts, comments, and fruit full advice.

\section{References}

[1] FAO, 200. The Energy and Agriculture Nexus. Environment and Natural Resources, Working Paper No. 4, Rome, pp. 15-21.

[2] World Energy Council, 2013. World Energy Resources. 2013 Survey, London, pp. 13-53.

[3] Sanchez, O., Cardona, C., 2007. Trends in biotechnological production of fuel ethanol from different feedstock: Review. Bioresource Technology 99, 3-9.

[4] Tesfaye, M., Gebru, Y., 2011. Assessment of Biofuel Development Activities in Ethiopia. Forum for Environment, Addis Ababa, Ethiopia, pp. 12-23.

[5] Anwar, Z., Gulfraz, M., Irshad, M., 2014. Agro-industrial lignocellulosic biomass a key to unlock the future bio-energy a brief review. Journal of radiation research and applied sciences $7,1-5$.

[6] Acosta, V., Paul, J., Ricana, M., Maceda, L., Aguinaldo, E., 2011. Application of Multi-criteria analysis in Management of Municipal Waste in Chosen Region in Poland. International Conference on Solid Waste, Hong Kong, pp. 56 -71.

[7] Bita, C. and Gerats, T., 2013. Plant tolerance to high temperature in a changing environment: scientific fundamentals and production of heat stress-tolerant crops. Frontiers in Plant Science, pp. 2-6

[8] Barber, R., Henningsson, M., Pamment, B., 2002. Acceleration of high gravity yeast fermentations by acetaldehyde addition. Biotechnology Letters 24, 891-895. 